\title{
Eficiência in vitro de acaricidas sobre carrapatos de bovinos no Estado do Rio Grande do Sul, Brasil
}

\author{
In vitro evaluation of acaricides efficiency to bovine's ticks of Rio Grande do Sul State, Brazil
}

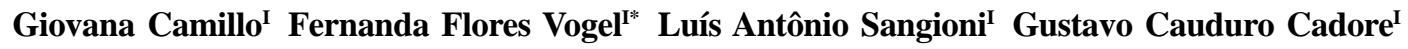 \\ Roberta Ferrari ${ }^{I}$
}

\section{RESUMO}

A infestação por carrapatos em bovinos é responsável por perdas econômicas significativas à indústria animal em várias regiões brasileiras, incluindo o Rio Grande do Sul. As perdas se devem ao stress, a perdas de peso e a injúrias na pele, bem como aos custos com tratamentos. O uso indiscriminado de carrapaticidas tem contribuído para o aparecimento da resistência genética dos ixodídeos a várias drogas, representando um sério problema no controle de carrapatos. O objetivo deste trabalho foi avaliar a susceptibilidade das diferentes espécies de campo de Rhipicephalus (Boophilus) microplus aos acaricidas usados nos controles dos parasitas. Para tanto, amostras de carrapato foram coletadas em 42 propriedades localizadas em diferentes municípios do Estado para a realização do teste de imersão de teleóginas (biocarrapaticidograma). A associação do amitraz e do clorpirifós resultou na droga que apresentou maior eficácia em $100 \%$ das propriedades testadas (11/11). Associações com cipermethrina-clorpirifós-citronelol foram eficientes nos carrapatos em $61 \%$ das propriedades (25/41) e cipermetrinaethion, em 37\% (10/27). A cipermetrina foi eficiente em $20,7 \%$ (6/29) e o amitraz, um dos produtos mais utilizados nas propriedades, foi eficiente em 14,2\% (6/42) das propriedades. Os resultados demonstraram que muitas drogas utilizadas no controle do $\boldsymbol{R}$. (B.) microplus no Estado apresentaram baixa eficácia, conforme os resultados dos testes in vitro. A baixa eficácia das drogas provavelmente se deve à resistência genética desenvolvida pelos parasitas devido ao uso indiscriminado dos carrapaticidas a campo.

Palavras-chave: Rhipicephalus (Boophilus) microplus, resistência parasitária, carrapato, bovinos, acaricidas.

\begin{abstract}
Infestation by ticks is responsible for significant economic losses to the cattle industry in several regions, including Rio Grande do Sul (RS) state. Losses may be derived from animal stress, weight loss and skin damage, as well as from costs with treatment. The indiscriminated use of pesticides against these parasites has resulted in high levels of genetic resistance to several drugs, representing a problem for disease control. Thus, the objective of this research was to evaluate the susceptibility of field specimens of Rhipcephalus (Boophilus) microplus to the acaricides used in the control of the parasite. Female ticks obtained from 42 farms in different counties of RS were submitted to the engorgeted female immersion test, to estimate the resistance of the parasite to the drugs. Amitraz in combination with chlorpyriphos was the most efficient drug, acting effectively in ticks from $100 \%$ of the tested farms (11/ 11). Associations of cypermethrin-chlorpyriphos-citronelol were efficient in ticks from $61 \%$ of the farms (25/41) and cypermethrin-ethion in 37\% (10/27). Cypermethrin alone was efficient in 20,7\% (6/29) and Amitraz. one of the most used products, was efficient in ticks from only 14,2\% (6/42) of the farms. These results demonstrate that most drugs used in the control of $\boldsymbol{R}$. B. microplus in the state present low efficacy, as measured by in vitro tests. The low drug efficacy is probably derived from the genetic resistance developed by the parasites due to the indiscriminated and inadequate use of these acaricides in the field.
\end{abstract}

Key words: Rhipicephalus (Boophilus) microplus, parasitary resistance, tick, cattle, acaricide.

'Departamento de Medicina Veterinária Preventiva (DMVP), Laboratório de Doenças Parasitárias (LADOPAR), Universidade Federal de Santa Maria (UFSM), Centro de Ciências Rurais (CCR). Av. Roraima, 1000, Camobi, 97105000, Santa Maria, RS, Brasil. E-mail: fervogel@smail.ufsm.br.*Autor para correspondência. 


\section{INTRODUÇÃO}

\section{O carrapato Rhipicephalus (Boophilus)}

microplus é responsável por grandes perdas econômicas na pecuária bovina, principalmente nas raças de origem européia, predominantes no Sul do Brasil. Este ácaro é de notada importância devido aos sérios prejuízos econômicos acarretados, como perdas na produção de leite, carne e couro, bem como por ser vetor de Anaplasma spp e de Babesia spp, agentes etiológicos da Tristeza Parasitária Bovina (CORDOVES, 1997). O Brasil gasta anualmente cerca de $R \$ 800$ milhões de reais com produtos químicos no tratamento de parasitas. O quadro atual do controle químico do carrapato se caracteriza pelo aumento progressivo do número de cepas resistentes aos principais acaricidas utilizados e, conseqüentemente, por um aumento na freqüência da aplicação (FURLONG et al., 2007). O controle do $\boldsymbol{R}$. (B.) microplus é baseado quase que exclusivamente na utilização de produtos químicos, principalmente por meio de antiparasitários que atuam por contato (NOLAN, 1994). Além disso, na maioria das vezes, o tratamento dos animais não é realizado por orientação de um médico-veterinário e não tem como base um tratamento estratégico que leva em consideração as condições epidemiológicas. O tratamento dos animais é realizado pelos próprios produtores, sem nenhuma orientação técnica.

A resistência do $\boldsymbol{R}$. (B.) microplus aos acaricidas frente a diferentes princípios ativos e em diferentes condições de manejo vem sendo relatada por vários pesquisadores . Na Austrália, por exemplo, em 1980, foi detectada uma cepa resistente ao amitraz, ao cyamizol e ao chlorometiuron (NOLAN, 1981). No Brasil, resistência do $\boldsymbol{R}$. (B.) microplus aos acaricidas foi relatada por pesquisadores frente aos diferentes grupos químicos de acaricidas e nas mais distintas regiões (LEITE et al. 1995; CAMPOS JÚNIOR \& OLIVEIRA, 2005).

Deve-se sempre escolher um produto comprovadamente eficaz e que determina uma melhor utilização do princípio ativo e a racionalização do manejo. Para isso, deve-se assegurar a inexistência de resistência do $\boldsymbol{R}$. (B.) microplus. Além disso, é necessário conhecer a dinâmica da resistência do $\boldsymbol{R}$. (B.) microplus frente aos acaricidas no Estado do Rio Grande do Sul. Nesse sentido, deve ser utilizado um teste de resistência aos acaricidas (biocarrapaticidograma), que é recomendado para a detecção da sensibilidade ou da resistência de teleóginas aos produtos carrapaticidas. Considera-se primordial a ação dos acaricidas sobre este estádio dos carrapatos. Assim, os resultados do biocarrapaticidograma são de aplicabilidade prática imediata, uma vez que a partir deles podem ser recomendadas alternativas mais eficientes de princípios ativos (CORDOVÉS, 1997).

A ineficiência no controle dos carrapatos por meio da aplicação de acaricidas continua sendo um desafio para as empresas, os pesquisadores e principalmente para os médicos veterinários. Este trabalho foi desenvolvido com intuito de monitorar o grau de eficiência de algumas classes de antiparasitários a cepas de $\boldsymbol{R}$. (B.) microplus coletadas em diferentes regiões do Rio Grande do Sul (RS).

\section{MATERIAL E MÉTODOS}

Para avaliação da eficiência de acaricidas frente a diferentes cepas de $\boldsymbol{R}$. (B.) microplus, foram utilizadas amostras de carrapatos provenientes de diferentes regiões do Estado do Rio Grande do Sul. Estas amostras foram utilizadas para testes in vitro, para detecção de susceptibilidade ou de resistência destas cepas frente a diferentes acaricidas.

Amostras de teleóginas foram coletadas de 42 propriedades criadoras de bovinos produtores de carne e/ou leite. Foram coletadas amostras de teleóginas provenientes dos municípios listados a seguir: Alegrete (1), Arroio Grande (3), Cacequi (1), Cachoeira do Sul (1), Dilermando de Aguiar (1), Erval Seco (1), Jóia (1), Lajeado (1), Nova Palma (2), Pantano Grande (2), Restinga Seca (1), Rio Pardo (1), Rosário do Sul (2), Santa Maria (8), Santa Rosa (1), Santiago (2), Santo Antônio das Missões (1), São Borja (2), São Gabriel (3), São Martinho da Serra (1), São Pedro do Sul (2), São Sepé (2), Toropi (1) e Tupaciretã (1), totalizando 24 municípios. Estes municípios compreendem as regiões: Central (13/24; 54,16\%), Planalto Centro-norte (2/24; 8,33), Noroeste (2/24; 8,33\%), Fronteira Oeste (6/24; $25 \%$ ) e Sul (1/24; 4,18\%) do RS. Não foram coletadas amostras de carrapato pertencentes às regiões Planalto Nordeste, Serrana, Litoral Norte, Metropolitana e grande Porto Alegre.

Foram testadas cinco bases químicas de maior utilização por parte dos produtores, geralmente as mais comercializados pelas casas agropecuárias. Os produtos testados foram: amitraz a 12,5\% (grupo químico das amidinas), diluído em água destilada na concentração final de 0,00006\%; cipermetrina a 15\% (piretróide), na concentração final de 0,00015\%; cipermetrina a $15 \%$ + clorpirifós a $25 \%$ + citronelol a 1\% (associação piretróide-organofosforado-citronelol), na concentração final de 0,00015\%, 0,00025\% e $0,00001 \%$, respectivamente; cipermetrina a $8 \%$ + ethion a 60\% (associação de piretróide-ethion), na 
concentração final de $0,00008 \%$ e $0,0006 \%$, respectivamente; e, por fim, a associação química amitraz a 50\% + clorpirifós a 50\% (amidinaorganofosforado), ambos na concentração final de $0,0005 \%$. Quanto às amidinas, quando sobravam teleóginas a serem testadas, foram utilizadas diferentes formulações comerciais. Assim, os resultados foram expressos na média de eficiência obtida a partir dos diferentes testes.

A coleta das teleóginas foi realizada manualmente em bovinos infestados de diferentes raças e em diferentes condições de manejo e, em seguida foram armazenadas e acondicionadas sob refrigeração a $4^{\circ} \mathrm{C}$. O tempo entre a coleta e a realização do teste não excedeu 48 horas. No Laboratório de Doenças Parasitárias da Universidade Federal de Santa Maria (UFSM), as teleóginas foram reavaliadas para o descarte das que se encontravam pouco ingurgitadas (tamanho inferior a 4mm) ou em estado de inércia, para então ser realizado o teste de imersão, também conhecido como biocarrapaticidograma, o qual foi desenvolvido por DRUMMOND et al. (1973). Os testes foram realizados em duplicata e os resultados foram expressos por meio da média dos valores obtidos em cada bateria. Para evitar interferência no teste, foi assegurado que os animais em que as amostras foram coletadas não haviam sido submetidos a tratamento com acaricidas em um intervalo inferior a 21 dias. Assim, foram utilizados como parâmetros a postura das teleóginas e o índice de eclodibilidade desses ovos. A eficiência legalmente aceitável para uma base química carrapaticida ser licenciada pelo Ministério da Agricultura deve ser igual ou superior a 95\% sobre uma cepa sensível de $\boldsymbol{R}$. (B.) microplus (MINISTÉRIO DAAGRICULTURA, 1987). O percentual de susceptibilidade é obtido quando os resultados são plotados na equação desenvolvida por DRUMMOND et al. (1973):

Eficiência Reprodutiva

$E R^{*}=\frac{\text { peso dos ovos }}{\text { peso das teleóginas }} \times \%$ eclosão $\times 20000 * *$

A constante 20.000 significa o número estimado de ovos em $1 \mathrm{~g}$ de ovos de $\boldsymbol{R}$. (B) microplus.

\section{Eficiência do Produto}

Os resultados obtidos foram submetidos à análise estatística pelo Teste de Kruskal -Wallis com significância de $5 \%(\mathrm{P}<0,05)$.

\section{RESULTADOS E DISCUSSÃO}

Os resultados obtidos estão apresentados nas tabelas 1,2 e 3 . Com base nos resultados, observouse observado que a resistência parasitária do $\boldsymbol{R}$. (B.) microplus aos acaricidas é um grande problema, uma vez que, em 42 das propriedades avaliadas, existe resistência a, pelo menos, um dos acaricidas testados (Tabelas 1 e 2). No entanto, a eficiência dos diferentes acaricidas frente às cepas de $\boldsymbol{R}$. (B.) microplus variou significativamente. Considerando as10 propriedades em que havia teleógenas suficientes para testar todas as formulações, em duas propriedades foi observada susceptibilidade apenas à associação de amitraz + clorpirifós e para as demais formulações testadas foi observada resistência do $\boldsymbol{R}$. (B.) microplus.

Pelos resultados da análise estatística, pode-se determinar que, quando os cinco grupos foram comparados conjuntamente, apresentaram diferenças estatísticas $(\mathrm{P}<0,05)$, porém, quando comparados grupo a grupo, somente o amitraz e a cipermetrina não diferiram estatisticamente.

Com relação ao amitraz, os índices de eficiência variaram entre 0 e $100 \%$. Em apenas seis propriedades (6/42 - 14,2\%;) foi observada sensibilidade do $\boldsymbol{R}$. (B.) microplus acima de $95 \%$. O fato de que em apenas seis propriedades testadas foi observada sensibilidade $\boldsymbol{R}$. (B.) microplus ao amitraz acima de 95\% era esperado. Este princípio ativo vem sendo mais utilizado pelos produtores rurais, o que aumenta a pressão de seleção, favorecendo o desenvolvimento das cepas resistentes ao princípio ativo. Além disso, na maioria das vezes, o tratamento é realizado de forma supressiva, o que predispõe ainda mais o aparecimento de resistência parasitária. Resultado semelhante foi encontrado por JONSSON \& HOPE (2007), os quais descrevem que o amitraz continua sendo um dos acaricidas mais populares no controle dos carrapatos nos bovinos, na Austrália, na América Latina e na África do Sul. O uso inadequado dos produtos de pulverização, no que se refere à subdosage ou à superdosage (BIANCHI et al., 2003), e a alta freqüência dos banhos carrapaticidas selecionam e propagam o alelo de resistência por pressão de seleção (FURLONG \& MARTINS, 2000).

A resistência ou a baixa susceptibilidade do R. (B.) microplus ao amitraz também foi registrada por FLAUSINO et al. (1995) no Rio de Janeiro, YAMAMURA \& MERLINI (1998) no Paraná e FURLONG (1999) em Minas Gerais, o que não foi verificado por SOUZA et al. (1999) e por JONSSON \& HOPE (2007), os quais citam que o amitraz foi o único acaricida eficaz em Santa Catarina e na Austrália, respectivamente. 
Tabela 1 - Grau de eficiência de acaricidas frente às cepas de Rhipicephalus (Boophilus) microplus coletadas em 20 propriedades diferentes, no Estado do Rio Grande do Sul.

\begin{tabular}{|c|c|c|c|c|c|}
\hline \multirow{2}{*}{ Propriedade } & \multirow[b]{2}{*}{$A m i * 1$} & \multirow[b]{2}{*}{ Ciper $^{2}$} & \multirow[b]{2}{*}{ Ciper + clor $^{3}$} & \multirow[b]{2}{*}{ Ciper + ethi ${ }^{4}$} & \multirow[b]{2}{*}{ Ami + clor } \\
\hline & & & & & \\
\hline 01 & $19 \%$ & NT & $100 \%$ & NT & NT \\
\hline 02 & $49 \%$ & NT & 0 & NT & NT \\
\hline 03 & $5,5 \%$ & NT & $100 \%$ & NT & NT \\
\hline 04 & $12,6 \%$ & NT & NT & NT & NT \\
\hline 05 & $39 \%$ & $88 \%$ & $100 \%$ & NT & NT \\
\hline 06 & 0 & $100 \%$ & $100 \%$ & NT & $100 \%$ \\
\hline 07 & $16,6 \%$ & $50 \%$ & 0 & $48 \%$ & $100 \%$ \\
\hline 08 & $100 \%$ & NT & $100 \%$ & $100 \%$ & NT \\
\hline 09 & 0 & NT & $100 \%$ & $75 \%$ & NT \\
\hline 10 & $48 \%$ & NT & $100 \%$ & $100 \%$ & NT \\
\hline 11 & $94 \%$ & $90 \%$ & $94,6 \%$ & $99 \%$ & NT \\
\hline 12 & $53 \%$ & $76 \%$ & $14 \%$ & $97 \%$ & NT \\
\hline 13 & 0 & NT & $95,7 \%$ & $60,8 \%$ & NT \\
\hline 14 & $62,7 \%$ & NT & $9,1 \%$ & NT & NT \\
\hline 15 & $67,6 \%$ & NT & $100 \%$ & NT & NT \\
\hline 16 & $92 \%$ & NT & $100 \%$ & NT & NT \\
\hline 17 & $100 \%$ & $67,4 \%$ & $100 \%$ & 0 & NT \\
\hline 18 & 74,25 & $100 \%$ & $100 \%$ & $90,6 \%$ & NT \\
\hline 19 & $78,3 \%$ & $100 \%$ & $75,8 \%$ & $100 \%$ & NT \\
\hline 20 & $57,4 \%$ & 0 & $37 \%$ & NT & NT \\
\hline
\end{tabular}

o grau de eficiência para este princípio foi calculado por meio da média de eficiência obtida de diferentes formulações comerciais. NT - amostra não testada. ${ }^{1}$ Amitraz, ${ }^{2}$ Cipermetrina, ${ }^{3}$ Clopirifós, ${ }^{4}$ Ethion.

Quanto à susceptibilidade à cipermetrina, foram testadas amostras provenientes de 29 propriedades, sendo que o índice de eficiência variou entre 0 e 100\%. Em 23 propriedades, o grau de eficiência frente ao $\boldsymbol{R}$. (B.) microplus foi inferior a 95\%. A eficiência média foi de $44,03 \%$. Esta eficiência média não surpreende, uma vez que os piretróides foram amplamente utilizados anteriormente, tanto para aspersão como em imersão e o caráter da resistência do R. (B.) microplus aos piretróides é irreversível. No Rio Grande do Sul, LARANJA et al. (1989) identificaram uma estirpe resistente a carrapaticidas piretróides (cipermetrina, decametrina, flumetrina e cialotrin). No Rio de Janeiro, LEITE (1988) detectou baixa eficiência dos piretróide frente ao $\boldsymbol{R}$. (B.) microplus. Além disso, LEITE et al. (1995) relataram que existe uma variação da eficiência entre os diferentes tipos de piretróides disponíveis comercialmente. No presente estudo, devese salientar que, embora a resistência do $\boldsymbol{R}$. (B.)microplus aos piretróides esteja amplamente distribuída nas 29 propriedades testadas, em seis a eficiência foi superior a 95\%.

A susceptibilidade do $\boldsymbol{R}$. (B.) microplus à associação de cipermetrina com organofosforado (cipermetrina + clorpirifós + citronelol) variou entre 0 e 100\%. Das 41 amostras, 60,9\% (25/41) apresentaram eficiência superior a 95\%. Outra associação de cipermetrina + ethion foi eficaz em 37\% (10/27) das propriedades em que foi avaliada, com os índices de eficiência variando entre 0 e 100\%. Já a associação de amitraz-organofosforado (amitraz + clorpirifós) foi $100 \%$ eficaz nas 11 propriedades analisadas.

Quanto à susceptibilidade do $\boldsymbol{R}$. (B.) microplus às associações de princípios ativos, a eficiência destas foi superior à eficiência obtida com os princípios não-associados. A média de eficiência das três associações testadas foi de $85,26 \%$, sendo que a menor susceptibilidade foi encontrada na associação de cipermetrina + ethion (72,5\%), enquanto que a associação de amitraz + clorpirifós foi $100 \%$ eficaz nas amostras testadas. Este fato provavelmente está relacionado à pressão de seleção imposta às cepas de $\boldsymbol{R}$. (B.) microplus, uma vez que a última associação é a mais recente no mercado e está disponível apenas para aspersão. No entanto, em algumas propriedades foi demonstrada a ineficiência da associação de cipermetrina + clorpirifós + citronelal e da associação de cipermetrina + ethion. Quanto à associação de 
Tabela 2 - Grau de eficiência de acaricidas frente às cepas de Rhipicephalus (Boophilus) microplus coletadas em 22 propriedades diferentes, no Estado do Rio Grande do Sul.

\begin{tabular}{|c|c|c|c|c|c|}
\hline \multirow{2}{*}{ Propriedade } & \multicolumn{5}{|c|}{ - Grau de eficiência frente ao R. (B.) microplus-- } \\
\hline & $\mathrm{Ami}^{* 1}$ & Ciper $^{2}$ & Ciper + clor $^{3}$ & Ciper + ethi ${ }^{4}$ & Ami + clor \\
\hline 21 & $100 \%$ & $100 \%$ & $91,5 \%$ & NT & NT \\
\hline 22 & $33,3 \%$ & 0 & 0 & 0 & $100 \%$ \\
\hline 23 & $42,1 \%$ & $67,5 \%$ & $86 \%$ & $91 \%$ & $100 \%$ \\
\hline 24 & $59,4 \%$ & $99,5 \%$ & $100 \%$ & $91,9 \%$ & $100 \%$ \\
\hline 25 & $27 \%$ & $68,2 \%$ & $100 \%$ & $85,9 \%$ & NT \\
\hline 26 & $99,9 \%$ & $99,5 \%$ & $85,2 \%$ & $92,3 \%$ & NT \\
\hline 27 & $100 \%$ & NT & $80 \%$ & NT & NT \\
\hline 28 & $27,2 \%$ & $9 \%$ & $99 \%$ & $43 \%$ & NT \\
\hline 29 & $51,4 \%$ & $20,1 \%$ & $100 \%$ & $100 \%$ & $100 \%$ \\
\hline 30 & $3,6 \%$ & 0 & $100 \%$ & $32 \%$ & $100 \%$ \\
\hline 31 & $49,3 \%$ & $23 \%$ & $100 \%$ & $100 \%$ & $100 \%$ \\
\hline 32 & $47,7 \%$ & $29 \%$ & $100 \%$ & $96 \%$ & NT \\
\hline 33 & $11,9 \%$ & 0 & $75,3 \%$ & 73,91 & NT \\
\hline 34 & $41,9 \%$ & 0 & $99,6 \%$ & $22,7 \%$ & NT \\
\hline 35 & $85 \%$ & 0 & $99 \%$ & $24 \%$ & NT \\
\hline 36 & $40,3 \%$ & 0 & $85,9 \%$ & $59,4 \%$ & NT \\
\hline 37 & $2 \%$ & 0 & $98 \%$ & NT & NT \\
\hline 38 & $29,8 \%$ & $41,6 \%$ & $100 \%$ & $98,1 \%$ & $100 \%$ \\
\hline 39 & $36,1 \%$ & $8,8 \%$ & $98,1 \%$ & $78 \%$ & $100 \%$ \\
\hline 40 & $66,6 \%$ & $39,4 \%$ & $83,2 \%$ & $99,2 \%$ & $100 \%$ \\
\hline 41 & $8,7 \%$ & NT & $100 \%$ & NT & NT \\
\hline 42 & $96,9 \%$ & 0 & $7,7 \%$ & NT & NT \\
\hline
\end{tabular}

o grau de eficiência para este princípio foi calculado por meio da média de eficiência obtida de diferentes formulações comerciais. NT - amostra não testada. ${ }^{1}$ Amitraz, ${ }^{2}$ Cipermetrina, ${ }^{3}$ Clopirifós, ${ }^{4}$ Ethion.

amitraz + clorpirifós, em 30\% das amostras testadas, as cepas de $\boldsymbol{R}$. (B.) microplus foram susceptíveis apenas a essa associação, mostrando-se resistentes aos demais princípios testados. Resultados semelhantes foram encontrados por CAMPOS JUNIOR \& OLIVEIRA (2005), em que a associação de piretróideorganofosforado (cipermetrina-diclorvos) na região de Ilhéus apresentou a maior eficiência média dos quatro produtos comerciais testados (75,73\%). OLIVEIRA (1999), em Minas Gerais (MG), e SANTANA(2001), em
Pernambuco (PE), observaram eficiências médias maiores das associações quando comparadas aos piretróides. A associação triclorfon-coumafós-cyflutrin apresentou uma eficiência média de 75,13\%. OLIVEIRA (1999) notificou 55,21\% de eficiência média ao coumafós em MG, SANTANA (2001) em PE registrou eficiências médias de $86,44 \%$ e $100,00 \%$ para o coumafós e a associação triclorfon-coumafós, respectivamente. Esses resultados demonstram que o organofosforado, quando associado ao amitraz ou ao

Tabela 3 - Susceptibilidade de 42 amostras de Rhipicephalus (Boophilus) microplus, coletadas em diferentes regiões do Estado do Rio Grande do Sul, frente a diferentes acaricidas testados.

\begin{tabular}{lcc}
\hline Acaricida & $\begin{array}{c}\text { Números de propriedades com cepas de } \boldsymbol{R} \text {. (B.) microplus } \\
\text { com eficiência igual ou superior a 95\% }\end{array}$ & Eficiência média (\%) \\
\hline Amitraz & $6 / 42-14,2 \%$ & $48,3\left(\mathrm{a}^{*}\right)$ \\
Cipermetrina & $6 / 29-20,6 \%$ & $44,0\left(\mathrm{a}^{*}\right)$ \\
Cipermetrina + clorpirifós+ citronelal & $25 / 41-60,9 \%$ & 80,8 (b) \\
Cipermetrina + ethion & $10 / 27-37 \%$ & $72,5(\mathrm{c})$ \\
Amitraz + clorpirifós & $11 / 11-100 \%$ & 100 (d) \\
\hline
\end{tabular}

*Não demonstram diferença estatística $(\mathrm{P}<0,05)$.

Ciência Rural, v.39, n.2, mar-abr, 2009. 
piretróide, pode ser utilizado com boa eficiência em um programa de controle estratégico ao carrapato.

Avaliando-se a eficiência média (Tabela 3) de cada um dos princípios testados, observou-se que a melhor média foi obtida com a associação de amitraz com clorpírifós $(100 \%)$ e a pior foi obtida com a cipermetrina (44,03\%)

\section{CONCLUSÃO}

Os resultados obtidos indicam que a resistência do $\boldsymbol{R}$. (B.) microplus aos acaricidas vem aumentando gradativamente tanto em número como em amplitude de princípios ativos. Menor susceptibilidade foi encontrada principalmente frente aos piretróides e às amidinas. As associações apresentaram eficiência média superior quando comparada a eficiência dos produtos não-associados, constituídos de amidina e deltametrina. Este fato salienta a necessidade de mais estudos acerca dos mecanismos de resistência parasitária, assim como de programas estratégicos para o controle do $\boldsymbol{R}$. (B.) microplus. Além disso, reafirma a importância da realização de um controle estratégico do carrapato com uso racional dos antiparasitários e com o monitoramento constante da eficiência do acaricida utilizado neste controle frente à cepa de $\boldsymbol{R}$. (B.) microplus em questão.

\section{AGRADECIMENTOS}

Os autores agradecem os professores: Luis Felipe Dias Lopes e Andréia Zanella, do Departamento de Estatística da Universidade Federal de Santa Maria, pela realização da análise estatística.

\section{REFERÊNCIAS}

BIANCHI, M.W. et al. Factors related to cattle infestation level and resistance to acaricides in Boophilus microplus tick populations in New Caledonia. Veterinary Parasitology, v.112, p.75-89, 2003.

CAMPOS JUNIOR, D.A.; OLIVEIRA, P.R. Avaliação in vitro da eficiência de acaricidas sobre Boophilus microplus (Canestrini, 1887) (Acari: Ixodidae) de bovinos no município de Ilhéus, Bahia, Brasil. Ciência Rural, v.35, p. 1386-1392,, 2005.

CORDOVÉS, C.O. Carrapato: controle ou erradicação. Porto Alegre: Guaíba Agropecuária, 1997. 197p.

DRUMMOND, R.O. et al. Boophilus annulatus and Boophilus microplus: laboratory tests for insecticides. Journal of Economic Entomology, v.66, p.130-133, 1973.

FAUSTINO, M.A.G. et al. Eficiência in vitro de produtos carrapaticidas em fêmeas ingurgitadas de cepas de Boophilus microplus da subregiãoda Zona da Mata de Pernambuco. Revista Brasileira de Parasitologia Veterinária, v.4, sup.1, p.58, 1995.
FURLONG, J. Diagnosis of the susceptibility of the cattle tick, Boophilus spp to acaricides in Minas Gerais State, Brazil. In: SEMINARIO INTERNACIONAL DE PARASITOLOGIA ANIMAL, 4., 1999, Puerto Vallarta, México. Proceedings... Puerto Vallarta : CONASAGA, 1999. p.41-46.

FURLONG, J.; MARTINS, J.R.S. Resistência dos carrapatos aos carrapaticidas. Juiz de Fora: CNPGL-EMBRAPA, 2000 25p. (Boletim Técnico 59).

FURLONG, J. et al. O carrapato dos bovinos e a resistência: temos o que comemorar? A Hora Veterinária, v.159, p.2632, 2007.

JONSSON, N.N., HOPE, M. Progress in the epidemiology and diagnosis of amitraz resistance in the cattle tick Boophilus microplus. Veterinary Parasitology, v.146, p.193-198, 2007.

LARANJA, R.J. et al. Identificação de uma estirpe de Boophilus microplus resistente a carrapaticidas piretróides no Estado do Rio Grande do Sul. In: SEMINÁRIO BRASILEIRO DE PARASITOLOGIA VETERINÁRIA, 6., 1989, Bagé. Anais... Bagé: CBPV, 1989. p.83.

LEITE, R.C. et al. In vitro susceptibility of engorged females from different populations of Boophilus microplus to commercial acaricides. Revista Brasileira de Parasitologia Veterinária, v.4, p.283-294, 1995.

LEITE, R.C. Boophilus microplus (Canestrini, 1887): susceptibilidade, uso atual e retrospectivo de carrapaticidas em propriedades das regiões fisiográficas da Baixada do Grande Rio e Rio de Janeiro. Uma abordagem epidemiológica. 1988. 151f. Tese (Doutorado em Parasitologia) - Universidade Federal Rural do Rio de Janeiro.

MINISTÉRIO DA AGRICULTURA. Normas para registros de parasiticidas de uso pecuário na Brasil. Brasília: Ministério da Agricultura, 1987. 19p.

NOLAN, J. Current developments in resistance to amidine and pyrethroid tickicides in Australia. In: WHITEHEAD,G.B.; GIBSON, J.D. Tick biology and control. Grahamstown: Rhodes University, 1981. p.109-114.

NOLAN, J. Acaricide resistence in the cattle tick Boophilus microplus. In: REPORT OF WORKSHOP LEADER NT FAO/ UN consultant, Porto Alegre, RS, Brazil. Abstract... Porto Alegre, 1994. p.21-25.

OLIVEIRA, P.R. Resistência do carrapato Boophilus microplus (Canestrini, 1887) (Acari: Ixodidae) a carrapaticidas em bovinos de leite da região da zona da mata de Minas Gerais. 1999. 33f. Dissertação (Mestrado em Ecologia Animal) - NT Universidade Federal de Juiz de Fora.

SANTANA, V.L.A. et al. Diagnóstico de situação do controle químico do carrapato dos bovinos (Boophilus microplus) em propriedades das mesorregiões da Mata e Agreste do Estado de Pernambuco - Brasil. Ciência Veterinária nos Trópicos, v.4, p.281-290, 2001.

SOUZA, A.P. et al.. Avaliação in vitro da eficiência de carrapaticidas no Estado de Santa Catarina. In: SEMINÁRIO BRASILEIRO DE PARASITOLOGIA VETERINÁRIA, 11, 1999, Salvador. Anais... Salvador: CBPV, 1999. p.72.

YAMAMURA, M.H.; MERLINI, L.S. Estudo in vitro da resistência de Boophilus microplus a carrapaticidas na pecuária leiteira do norte do Estado do Paraná. Semina, v.19, p.38-43, 1998. 